\title{
The effect of number of attribute-values in conjunctive concept identification
}

FRANK L. SLAYMAKER and IRWIN D NAHINSKY, University of Missouri, Colum bia, Mo. 65201

$A$ factorial experiment in conjunctive concept identification was performed in which number of attributes and number of attribute values were varied. Only number of attributes had a significant effect upon hypothesis sampling behavior, a finding which supports the notion that Ss sample only hypotheses compatible with positive instances as they are presented.

A number of theorists have hypothesized that $\mathrm{Ss}$ in a concept identification (CI) task sample randomly from a population of all possible hypotheses determined by the stimulus set and the specified rule (see Restle, 1962; Brower \& Trabasso, 1964) Since possible hypotheses vary with number of stimulus attributes as well as with number of values assumed by the attributes, the above theorists would predict that task difficulty would vary with both of these variables. Nahinsky \& McGlynn (1968) have presented evidence that $S$ s sample hypoth eses randomly from a set consistent with a positive instance when presented. Increasing the number of attributes increases the number of hypotheses consistent with each instance. Hence the set of hypotheses consistent with a positive instance is increased by increasing the number of attributes, and task difficulty is increased even if $\mathbf{S}$ samples hypotheses compatible with positive instances alone.

An increase in the number of attribute values would not produce an increase in hypotheses compatible with a single stimulus. Consequently, if Ss sample hypotheses only compatible with positive instances upon presentation, number of attribute values should not retard the sampling process. This study was designed to investigate the above relationships in a conjunctive $\mathrm{CI}$ task.

\section{METHOD}

The Ss were 180 students whose participation fulfilled an introductory psychology course requirement. The task was a two-category conjunctive $\mathrm{CI}$ task in which the correct classification rule depended upon the concept "striped circles." The stimulus sets varied in two ways: (a) the number of attributes $-3,4$, or 5 , and (b) the number of values -2 or 3 . This yielded six 30-S experimental conditions identified as: $3-2,3-3,4-2,4-3,5-2,5-3$, the first number in each pair indicating the number of attributes, the second the number of values.

Instructions about the task were very explicit and similar to those used by Levine (1966). Throughout the experimental session $S$ had for inspection a diagram displaying all possible stimuli that he could be presented. Each $\mathrm{S}$ was given a practice problem prior to the test problem to familiarize him with the procedure.

A blank-trial technique of the form used by Nahinsky \& Slaymaker (in press) was used. No feedback was presented on blank-trial stimuli and $\mathrm{Ss}$ were informed that this lack of feedback indicated nothing about the correctness of their responses.

The protocol consisted of an initial positive instance followed by three blanktrial stimuli each overlapping the initial instance by exactly two values (one hypothesis), one of which stimuli contained the correct cue combination. Three feed back trials followed, one of which contained the correct cue combination, all other stimuli having no value overlap with the initial positive instance. The same three blank-trial stimuli were again presented in random order. Each $S$ was then asked to guess the concept. According to an information analysis of the problem, only two positive instances are required to identify the concept.

The Ss were assigned to the six conditions in rotation according to the order in which they were scheduled.

\section{RESULTS AND DISCUSSION}

To measure the effect of increasing the number of values on sampling behavior of Ss, $\chi^{2}$ tests comparing the distributions of number of blank-trial stimuli sampled were done. Ss sampling two or three blank-trial stimuli were pooled to yield adequate expected frequencies. Comparisons were made between distributions for values within the same number of attributes since conditions differed over attributes with regard to the number of hypotheses consistent with the initial instance. Since only three blank-trial stimuli were used in each condition regardless of the number of potential hypotheses, an overall analysis would be biased across attributes because the relative proportion of compatible hypotheses presented decreased as attributes increased. All $\chi^{2}$ values were less than 1. For five attributes, $\chi^{2}(2)=.077, p>.95$ for four attributes, $\chi^{2}(2)=.119, p>.90$; for three attributes, $\chi^{2}(2)=.495, p>.75$. Thus, an increase in values has no significant effect upon hypothesis sampling behavior.

The conditions involving four and five attributes entailed blank-trial presentation of only some subset of stimuli corresponding to hypotheses compatible with the initial positive instance. Greater exposure of these combinations may have increased the likelihood of their subsequent selection. An analysis of protocols for nonlearning Ss provided information about such a blank. trial effect. A tabulation was made of Ss who failed to learn the concept but guessed a hypothesis consistent with the initial positive instance and one of the initial blank-trial stimuli.

For four-attribute stimuli there are six possible hypotheses, three of which were presented in the blank-trial series and one of which was correct. Therefore, of the remaining five hypotheses, two should be sampled at a rate greater than the expected .40. For Condition 4-2 the observed proportion for these hypotheses was .45 , and for Condition 4-3 it was .38.

For five attribute stimuli there are 10 possible hypotheses. By the above reasoning, two out of nine should be sampled at a rate greater than .22 if blank-trial presentations are to show any effect. For Condition 5.2 such hypotheses were sampled $19 \%$ of the time, and for Condition 5-3 the rate was .21 . For pooled data, both the expected and observed proportion of Ss sampling hypotheses consistent with the initial positive instance and one initial blank-trial stimulus equalled .311 . If blank-trial stimuli have some effect upon sampling behavior, it does not appear in the protocols of nonlearning Ss.

Since no significant effect upon sampling behavior was found for number of attribute values, data within attributes were pooled to test whether number of attributes had a significant effect upon hypothesis sampling behavior. A 3 by 3 factorial table was constructed with number of attributes: three, four, or five against number of blank-trial stimuli classified as representative of the concept; zero, one, or two or three. The mean number of stimuli so classified were: for five attributes, 0.72 ; for four attributes, 1.08; and for three attributes, 1.13. A $\chi^{2}$ test of association proved significant $\left[\chi^{2}(4)=15.33\right.$, $p<.005]$, indicating that number of attributes has a definite effect upon S's sampling behavior.

The data appear to substantiate the hypotheses set forth in the introduction. The number of attributes within each stimulus had a significant effect upon hypothesis sampling behavior. The data indicated further that blank-trial stimulus 
presentation had little effect upon hypothesis sampling behavior.

\section{REFERENCES}

BOWER. G.. \& TRABASSO, T. Concept identification. In R. C. Atkinson (Ed.), Studies in mathematical psycholog: Stanford: Stanford Lniversity Press, 1964. Pp. 32-96.

LEVINE, M. Hypothesis behavior by humans during discrimination learning. Journal of Experimental Psychology, 1966, 71, 331-338.
NAHINSKY. I. D.. \& MOCLYNN, F, D Hypothesis sampling in conjunctive concept identification. Psychonomic Science, 1968, 11 , 77-78.

NAHINSKY, I. D., \& SLAYMAKER, F. L. Sampling without replace ment and information processing following correct responses in concept identification. Journal of Experimental Psychology, in press.

RESTLE, F. The selection of strategies in cue learning. Psychological Review, 1962, 69, 329-343. ing behaviors of normals be adequate for the description of the behavior of the disadvantaged?

The purpose of the present study was to explore some of these effects of social influence on the disadvantaged as compared to normal Ss. The experimental design was a factorial analysis of variance with educational experience of the Ss, nature of the influencing source, and task difficulty as independent variables. Yielding to social influence was the dependent variable. The effects of primary concern were the main effect which contrasted normal and disadvantaged Ss and the Ss by task difficulty interaction.

\section{SUBJECTS}

The $S s$ were 48 students from the University of South Florida. Twenty-four of these Ss (12 males and 12 females) were regularly enrolled college students with a mean age of 19 . The other $24 \mathrm{Ss}$ ( 12 males and 12 females) were members of the High School Equivalency Program (HEP) at the university, also with a mean age of 19 . The HEP was designed to assist school drop-outs of migrant or farm worker background in reaching an educational level equivalent to that certified by the high school diploma. Thus, the two samples were similar in age and sex but different in educational, social, and economic respects associated with dropping out of school and being socialized within a migrant worker environment. While the particulars of these differences remained vague, it was assumed that the sample of HEP Ss, as compared to the sample of regular college $\mathrm{Ss}$, was educationally, socially, and economically disadvantaged.

\section{PROCEDURE}

Observations of socially influenced behavior were made by means of the Personal Opinions Inventory (POI), constructed and standardized by McDavid (1965) as a research instrument to efficiently measure conformity to attributed sources of influence. This paper and pencil device presented 30 statements of fact (easy and difficult) to the $S$ with an instruction for him to indicate his agreement or disagreement with each statement. Social influence was provided by informing the $S$ on each statement of the judgment of the majority of persons having been questioned previously. The specific nature of the social influence was transmitted in written instructions identifying the persons composing the reported majority. The POI consisted of standardized critical items on which the majority had indicated an incorrect judgment, interspersed with filler items on which the majority had expressed an accurate judgment. A conforming response was scored when the $S$ agreed with the majority on a critical item instead of 